\title{
Alchemilla vulgaris effects on egg production and quality expressed by heat- stressed quail during the late laying period
}

\author{
F. Akdemir ${ }^{1}$, A. Köseman ${ }^{2}$ \& I. Şeker ${ }^{3 \#}$ \\ ${ }^{1}$ Department of Nutrition, Faculty of Fisheries, Malatya Turgut Ozal University, 44210, Malatya, Turkey \\ ${ }^{2}$ Department of Crop and Animal Production, Akcadag Vocational School, Malatya Turgut Ozal University, 44210, \\ Malatya, Turkey \\ ${ }^{3}$ Department of Veterinary Zootechny, Faculty of Veterinary Medicine, Firat University, 23119, Elazig, Turkey
}

(Received 29 March 2019; Accepted 5 July 2019; First published online 14 October 2019)

\begin{abstract}
Copyright resides with the authors in terms of the Creative Commons Attribution 4.0 South African License.
See: http://creativecommons.org/licenses/by/4.0/za

Condition of use: The user may copy, distribute, transmit and adapt the work, but must recognize the authors and the South African Journal of Animal Science.
\end{abstract}

\begin{abstract}
Potential for mitigating effects of heat stress through dietary Alchemilla vulgaris (AV) supplementation during the late laying period of Japanese quail (Coturnix coturnix japonica) were investigated. A $2 \times 3$ factorial arrangement of environmental temperature $(E T)$ regimes and levels of dietary supplementation with $\mathrm{AV}(0 \%, 1 \%$, and $3 \%)$ was used in a 75 -day experiment. Twenty-five quail were randomly assigned to each treatment with five replicate cages of five birds. The birds were housed in temperature-controlled rooms at 22 $\pm 2{ }^{\circ} \mathrm{C}$ for $24 \mathrm{~h} /$ day $(\mathrm{TN})$ or $34 \pm 2{ }^{\circ} \mathrm{C}$ between $09 \mathrm{~h} 00$ and $17 \mathrm{~h} 00$ followed by $22 \pm 2{ }^{\circ} \mathrm{C}$ for $16 \mathrm{~h} /$ day (HS). The interaction of ET and supplement regimes was rarely significant. In HS quail supplemented with $1 \% \mathrm{AV}$, egg production was reduced and FCR was increased compared with the other treatments. Dietary AV was found to reduce egg production in TN conditions, but 3\% AV supplementation in the HS group prevented decreased egg production and improved FCR. Various indicators of egg quality were significantly affected by supplementation with AV at certain times during the experiment. Most effects of HS on egg quality were manifest in the first 15 days of ET regimes. Although HS significantly decreased eggshell weight until 31-45 days, AV supplementation improved it on the 45th day and then maintained it through the end of the experiment. Thus, AV may mitigate some effects of HS by partially preventing decreased egg production and increased FCR during the late laying period of Japanese quail.
\end{abstract}

Keywords: Coturnix coturnix japonica, flavonoids, supplement

\#Corresponding author: iseker@firat.edu.tr

\section{Introduction}

Japanese quail (Coturnix coturnix japonica) are preferred as laboratory animal models owing to their productivity per unit area and the ease and low cost of their care and feeding (Ruskin, 1991; Baer et al., 2015). They consume less feed than other poultry species, and produce large eggs in proportion to their weight. Their age at first egg is 40-42 days, and they reach maximum production after 4-5 weeks (Reddish et al., 2003). Quail eggs are rich in protein, minerals and carbohydrates (Tunsaringkarn et al., 2013). Their positive contribution to human nutrition and health enhances their utility as a food of animal origin in an adequate balanced diet, particularly in poor communities (Ndlovu, 2010).

It is commonly thought that living conditions of human and animals will deteriorate due to the increase in global warming in the near future. Animal foods play an important role in human nutrition, and production under these conditions may not be sufficient to satisfy needs. For animals, the primary result of increased ETs is oxidative stress. Environmental temperatures that exceed the thermo-neutral zone $\left(16-25{ }^{\circ} \mathrm{C}\right)$ of poultry cause significant yield (eggs and meat) losses, impaired egg quality, increased mortality rates, and low profitability (Lu et al., 2007; Sahin et al., 2008a, b; Akdemir et al., 2015). In recent years, there has been an increase in interest in the use of natural herbal additives to mitigate heat stress in the production of foods of animal origin (Leeson, 1986; Shane, 1988).

One of the perennial herbs is Alchemilla vulgaris (AV) or lady's mantle, a medicinal plant and member of the Rosaceae (rose) family, which has strong antimicrobial, antioxidant, and anticancer activities that have 
been attributed to their high levels of flavonoids, flavanol, tannins, anticianidins, saponines, and phenolic acid (Kiselova et al., 2006; Surai, 2013; Neagu et al., 2015). Flavonoids, which are regarded as the major ingredient of AV, have multiple biological influences, including antidiarrhoeal, antiarthritic, diuretic, depurative, and intestinal antiseptic effects, and improve menopausal distortions (Kahkonen et al., 1999; Galati \& O'Brien, 2004; Spiridonov et al., 2005; Nair et al., 2006). These beneficial effects of flavonoids (9.8 $\mathrm{mg} / \mathrm{g}$ dried whole plant) are attributed mainly to the activation of antioxidant enzymes (D'Agostino et al., 1998; Oktyabrskay et al., 2009), scavenging of free radicals (Hanasaki et al., 1994) and chelation of metal ions (Afanas'ev et al., 1989; Rice-Evans et al., 1997). Although there have been many studies on the use of $A V$ in the field of biological and metabolic disturbances, no studies have been found on the effects of $A V$ on the laying performance and egg quality of quails that have been exposed to high environmental temperatures. Therefore, the aim of the present study was to determine the effects of dietary AV supplementation on egg production and egg quality parameters during the late laying period of heat-stressed quail.

\section{Materials and Methods}

The experiment was approved (approval document no 2018/A-22) by the Committee on Animal Research at Inonu University, Malatya, Turkey. Birds $(N=150)$ at 20 -weeks old were purchased from a commercial company (Insanay Kanatli Hayvan Uretim Paz. Tic. Inc., Elazig, Turkey). They had an initial average bodyweight of $197.8 \pm 2.3 \mathrm{~g}$ (mean $\pm \mathrm{SE}$ ). The birds were randomly assigned to one of six treatments of 25 birds each. For each treatment there were five replicate cages of five birds per cage. The experiment spanned 75 days after an adaptation period of 10 days. The birds were housed in temperaturecontrolled rooms at $22 \pm 2{ }^{\circ} \mathrm{C}$ for $24 \mathrm{~h} /$ day (TN) or $34 \pm 2{ }^{\circ} \mathrm{C}$ for $8 \mathrm{~h} /$ day (between $09 \mathrm{hO0}$ and 17h00), followed by $22 \pm 2{ }^{\circ} \mathrm{C}$ for $16 \mathrm{~h} /$ day (HS) throughout the experiment. In both environmental conditions, the quail were fed one of three diets (Table 1), namely a basal diet or the basal diet supplemented with $1 \%$ or $3 \%$ of Alchemilla vulgaris in powder form (Altinterim Co., Elazig, Turkey). They were housed in cages that provided $100-120 \mathrm{~cm}^{2}$ floor area per bird. Birds were exposed to a diurnal cycle of 16 hours of light and 8 hours of darkness. Diets and fresh water were offered ad libitum throughout the experiment.

Table 1 Ingredients and nutrient composition of the basal diet (\%) $)^{1}$ fed to Japanese quail during the late laying period

\begin{tabular}{|c|c|}
\hline Ingredient & Amount (\%) \\
\hline Corn & 54.34 \\
\hline Soybean meal & 28.91 \\
\hline Soy oil & 4.96 \\
\hline Salt & 0.31 \\
\hline DL-methionine & 0.19 \\
\hline Limestone & 9.26 \\
\hline Dicalcium phosphate & 1.68 \\
\hline Vitamin and mineral premix ${ }^{2}$ & 0.35 \\
\hline \multicolumn{2}{|l|}{ Nutrient composition (\%, dry matter basis) } \\
\hline Crude protein & 18.09 \\
\hline Calcium & 3.73 \\
\hline Phosphorus & 0.63 \\
\hline Methionine $^{3}$ & 0.42 \\
\hline Lysine $^{3}$ & 1.04 \\
\hline Calculated metabolizable energy (Jurgens, 1996), kcal/kg & 2912 \\
\hline
\end{tabular}


Chemical analyses of the basal diet for crude protein (988.05), ether extract (932.06), crude fibre (962.09), crude ash (936.07), Ca (968.08), and $\mathrm{P}$ (965.17) were done in triplicate using the methods described by the AOAC International (1990). Energy and amino acid (methionine and lysine) ingredients were computed from tabular values for the feedstuffs (Jurgens, 1996).

Feed consumption, egg production and egg weight were measured on the 25th, 50th and 75th days of the experiment. In addition, egg weight, yolk weight, yolk colour, shell weight, shell thickness and Haugh unit were measured on the 15th, 30th, 45th, 60th, and 75th days of the experiment using two random eggs from each of the five replicates (total 10 eggs) per treatment for all measurements. The Roche colour fan was used to determine the egg yolk colour according to the CIE standard colorimetric system (F. Hoffman-La Roche Ltd., Basel, Switzerland). Haugh units were computed, following Eisen et al. (1962), as:

$$
\text { Haugh unit }=100 \log \left(\mathrm{H}+7.57-1.7 \mathrm{~W}^{0.37}\right)
$$

Where: $\mathrm{H}=$ albumen height $(\mathrm{mm})$

$\mathrm{W}=$ egg weight $(\mathrm{g})$ after determining albumen height by using a micrometre (TLM-N1010, Saginomiya, Tokyo, Japan)

A dial pipe gauge (Mitutoyo, 0.01-20 mm, Tokyo, Japan) was used to measure the eggshell thickness. Measurements were taken at the three regions of the shell (air cell, equator and sharp end) and averaged.

Data were analysed by two-way ANOVA using the PROC GLM procedure of SPSS (version 22.0 for Windows, Chicago, Illinois, USA). The model was:

$$
y_{i j k}=\mu+E_{i}+S_{j}+E x S_{i j}+e_{i j k}
$$

where: $y=$ response variable

$\mu=$ population mean

$\mathrm{E}=$ environmental temperature treatment

$\mathrm{S}=\mathrm{AV}$ supplementation treatment

$\mathrm{e}=$ residual error $\left[\operatorname{NID}\left(0, \sigma_{e}^{2}\right)\right]$

Differences among the treatment means were evaluated by the Duncan multiple comparison test. Differences were declared significant at $P<0.05$.

\section{Results}

Egg production, feed consumption, and egg weight were reduced, while FCR was increased for quail that were exposed to HS (Table 2). Supplementation with AV did not affect the performance of heat-stressed quail. However, egg production of the quail kept in TN conditions was reduced significantly by dietary AV supplementation $(P<0.05)$. However, AV supplementation at $1 \%$ of the diet contributed to increased FCR, while AV supplementation at $3 \%$ of diet significantly reduced FCR in the quails exposed to HS $(P<0.05)$.

From day 1 to 15, the shell weight, egg yolk height, albumen width and egg yolk index were all changed as a result of the heat stress $(P<0.05)$, while AV supplementation significantly changed the albumen and egg yolk height, albumen index and Haugh units (HU) (Table 3).

From 16 to 30 days, the egg weight, shell weight, albumen height and length and HU were significantly changed by heat stress $(P<0.05)$, and shell weight was significantly changed by AV supplementation and ET x AV level interaction effects (Table 4).

Heat stress was of little consequence for any of the traits that characterized the eggs in days 31 to 45 (Table 5), but shell weight, albumen height and length, egg yolk diameter and index were significantly affected by AV supplementation $(P<0.05)$.

Albumen height and $\mathrm{HU}$ were affected by heat stress $(P<0.05)$ from days 46 to 60 . Albumen height, egg yolk colour and HU were changed by AV supplementation $(P<0.05)$ during this period as well. The interaction effect of ET $\times$ AV from 46 to 60 days affected $\mathrm{HU}(P<0.05$, Table 6$)$.

In the last 15 days, albumen length was changed with AV supplementation $(P<0.05)$, and egg yolk colour was significantly affected by the ET x AV interaction $(P<0.05$, Table 7$)$. 
Table 2 Treatment means ( \pm SE) for effects of dietary supplementation with Alchemilla vulgaris and heat stress on performance in late laying period of quail

\begin{tabular}{|c|c|c|c|c|c|c|c|}
\hline \multirow{2}{*}{\multicolumn{2}{|c|}{ Temperature regime }} & \multicolumn{6}{|c|}{ Treatments $^{1}$} \\
\hline & & \multicolumn{3}{|c|}{ Thermoneutral } & \multicolumn{3}{|c|}{ Heat stress } \\
\hline \multicolumn{2}{|c|}{ Supplementation level } & 0 & 1 & 3 & 0 & 1 & 3 \\
\hline Variables $^{2}$ & Days & & & & & & \\
\hline \multirow[t]{2}{*}{ FBW, g } & & $216.0 \pm 3.6$ & $220.0 \pm 7.5$ & $209.0 \pm 6.3$ & $218.9 \pm 6.3$ & $221.5 \pm 8.8$ & $211.9 \pm 6.3$ \\
\hline & $1-25$ & 26.0 & 25.5 & 23.9 & 25.0 & 23.4 & 24.9 \\
\hline \multirow{4}{*}{$\begin{array}{l}\text { Feed intake, } \\
\text { g/d }\end{array}$} & $26-50$ & 34.0 & 25.8 & 30.1 & 28.5 & 28.2 & 30.2 \\
\hline & $51-75$ & 25.9 & 21.7 & 25.5 & 24.2 & 26.4 & 23.8 \\
\hline & $1-75$ & $28.6 \pm 2.7$ & $24.3 \pm 1.3$ & $26.5 \pm 1.9$ & $25.9 \pm 1.3$ & $26.0 \pm 1.4$ & $26.3 \pm 2.0$ \\
\hline & $1-25$ & 10.83 & 10.53 & 9.63 & 9.95 & 10.12 & 10.46 \\
\hline \multirow{4}{*}{ Egg weight, $g$} & $26-50$ & 9.89 & 11.21 & 10.02 & 10.00 & 10.22 & 9.93 \\
\hline & $51-75$ & 9.90 & 10.55 & 9.92 & 9.10 & 10.25 & 9.72 \\
\hline & $1-75$ & $10.21 \pm 0.3$ & $10.76 \pm 0.2$ & $9.86 \pm 0.1$ & $9.68 \pm 0.3$ & $10.20 \pm 0.1$ & $10.04 \pm 0.2$ \\
\hline & $1-25$ & 49.8 & 51.9 & 49.7 & 46.9 & 27.4 & 52.1 \\
\hline \multirow{4}{*}{$\begin{array}{l}\text { Egg } \\
\text { production, \% }\end{array}$} & $26-50$ & 80.0 & 38.1 & 44.3 & 64.3 & 32.8 & 70.8 \\
\hline & $51-75$ & 67.9 & 52.7 & 56.8 & 69.7 & 25.6 & 56.8 \\
\hline & $1-75$ & $65.9^{\mathrm{a}} \pm 8.9$ & $47.6^{a} \pm 4.7$ & $50.3^{a} \pm 3.6$ & $60.3^{a} \pm 6.9$ & $28.6^{b} \pm 2.2$ & $59.9^{a} \pm 5.6$ \\
\hline & $1-25$ & 4.83 & 4.67 & 4.99 & 5.36 & 8.44 & 4.57 \\
\hline \multirow{3}{*}{ FCR } & $26-50$ & 4.30 & 6.04 & 6.78 & 4.43 & 8.41 & 4.30 \\
\hline & $51-75$ & 3.85 & 3.90 & 4.53 & 3.82 & 10.06 & 4.31 \\
\hline & $1-75$ & $4.33^{\mathrm{a}} \pm 0.3$ & $4.87^{\mathrm{a}} \pm 0.6$ & $5.43^{\mathrm{a}} \pm 0.7$ & $4.54^{\mathrm{a}} \pm 0.5$ & $8.97^{b} \pm 0.6$ & $4.39^{\mathrm{a}} \pm 0.1$ \\
\hline
\end{tabular}

\footnotetext{
${ }^{1}$ Thermoneutral: housed in temperature-controlled rooms at $22 \pm 2{ }^{\circ} \mathrm{C}$ for $24 \mathrm{~h} /$ day, Heat stress: $34 \pm 2{ }^{\circ} \mathrm{C}$ for $8 \mathrm{~h} / \mathrm{d}$ ay (between $09 \mathrm{h00}$ and $17 \mathrm{~h} 00$ ) and $22 \pm 2{ }^{\circ} \mathrm{C}$ for $16 \mathrm{~h} /$ day, 0 : unsupplemented, 1 : supplemented with $1 \%$ Alchemilla vulgari powder, 3: supplemented with 3\% Alchemilla vulgari powder

${ }^{2}$ FBW: final bodyweight, FCR: feed conversion ratio, i.e. feed consumed (g)/eggs produced (g)

${ }_{a, b}$ Within a row, means with a common superscript do not differ with a probability of $P \leq 0.05$
} 
Table 3 Treatment means $( \pm S E)$ for effects of dietary supplementation with Alchemilla vulgaris and heat stress on egg quality traits that were expressed in late laying period during days 1 to 15 after the imposition of treatments

\begin{tabular}{|c|c|c|c|c|c|c|c|c|c|c|}
\hline \multicolumn{2}{|c|}{ Treatments $^{1}$} & $\mathrm{n}$ & $\begin{array}{c}\text { Egg } \\
\text { weight } \\
\text { (gr) }\end{array}$ & $\begin{array}{c}\text { Eggshell } \\
\text { weight } \\
\text { (gr) }\end{array}$ & $\begin{array}{l}\text { Eggshell } \\
\text { thickness } \\
\left(10^{-2} \mathrm{~mm}\right)\end{array}$ & $\begin{array}{l}\text { Albumen height } \\
\qquad(\mathrm{mm})\end{array}$ & $\begin{array}{l}\text { Albumen } \\
\text { length } \\
(\mathrm{mm})\end{array}$ & $\begin{array}{c}\text { Egg yolk } \\
\text { height } \\
(\mathrm{mm})\end{array}$ & $\begin{array}{l}\text { Egg yolk } \\
\text { diameter } \\
(\mathrm{mm})\end{array}$ & $\begin{array}{l}\text { Egg } \\
\text { width } \\
(\mathrm{mm})\end{array}$ \\
\hline \multicolumn{2}{|l|}{ TN } & 30 & $10.22 \pm 0.17$ & $0.91 \pm 0.02$ & $29.34 \pm 0.58$ & $4.07 \pm 0.20$ & $43.85 \pm 0.57$ & $10.44 \pm 0.19$ & $23.31 \pm 0.43$ & $24.49 \pm 0.16$ \\
\hline \multicolumn{2}{|l|}{ HS } & 30 & $10.03 \pm 0.15$ & $0.86 \pm 0.02$ & $27.95 \pm 0.64$ & $3.88 \pm 0.18$ & $42.35 \pm 0.76$ & $9.52 \pm 0.09$ & $22.90 \pm 0.33$ & $24.24 \pm 0.11$ \\
\hline & 0 & 10 & $10.25 \pm 0.24$ & $0.89 \pm 0.02$ & $29.23 \pm 0.96$ & $4.39^{c} \pm 0.52$ & $44.11 \pm 1.10$ & $10.70^{b} \pm 0.46$ & $22.78 \pm 0.54$ & $24.75 \pm 0.20$ \\
\hline \multirow[t]{3}{*}{ TN } & 1 & 10 & $10.40 \pm 0.39$ & $0.93 \pm 0.04$ & $29.82 \pm 1.01$ & $4.01^{\mathrm{ab}} \pm 0.30$ & $42.83 \pm 1.17$ & $10.68^{b} \pm 0.33$ & $23.43 \pm 0.28$ & $24.62 \pm 0.36$ \\
\hline & 3 & 10 & $9.99 \pm 0.26$ & $0.91 \pm 0.02$ & $28.96 \pm 1.11$ & $3.80^{\mathrm{ab}} \pm 0.15$ & $44.62 \pm 0.58$ & $9.95^{\mathrm{a}} \pm 0.08$ & $23.72 \pm 1.18$ & $24.09 \pm 0.25$ \\
\hline & 0 & 10 & $10.03 \pm 0.30$ & $0.87 \pm 0.04$ & $26.83 \pm 1.13$ & $4.16^{c} \pm 0.27$ & $40.21 \pm 1.16$ & $9.53^{\mathrm{a}} \pm 0.12$ & $22.53 \pm 0.51$ & $23.95 \pm 0.20$ \\
\hline \multirow[t]{3}{*}{ HS } & 1 & 10 & $9.83 \pm 0.21$ & $0.85 \pm 0.02$ & $28.75 \pm 1.01$ & $4.34^{c} \pm 0.25$ & $42.43 \pm 1.34$ & $9.31^{\mathrm{a}} \pm 0.15$ & $22.81 \pm 0.55$ & $24.17 \pm 0.11$ \\
\hline & 3 & 10 & $10.24 \pm 0.30$ & $0.86 \pm 0.03$ & $28.27 \pm 1.21$ & $3.13^{a} \pm 0.29$ & $44.41 \pm 1.20$ & $9.71^{a} \pm 0.16$ & $23.35 \pm 0.66$ & 24. $59 \pm 0.22$ \\
\hline & & & $\begin{array}{l}\text { Egg length } \\
(\mathrm{mm})\end{array}$ & $\begin{array}{c}\text { Shape index } \\
(\%)\end{array}$ & $\begin{array}{l}\text { Egg yolk } \\
\text { colour }\end{array}$ & $\begin{array}{l}\text { Albumen width } \\
\qquad(\mathrm{mm})\end{array}$ & $\begin{array}{l}\text { Egg yolk } \\
\text { Index (\%) }\end{array}$ & $\begin{array}{l}\text { Albumen } \\
\text { index (\%) }\end{array}$ & Haugh units & $\begin{array}{l}\text { Shell strength } \\
\left(\mathrm{kg} / \mathrm{cm}^{2}\right)\end{array}$ \\
\hline \multicolumn{2}{|l|}{ TN } & 30 & $30.87 \pm 0.23$ & $79.40 \pm 0.54$ & $4.93 \pm 0.19$ & $34.42 \pm 0.71$ & $45.08 \pm 0.98$ & $10.49 \pm 0.59$ & $87.76 \pm 1.15$ & $2.36 \pm 0.01$ \\
\hline \multicolumn{2}{|l|}{ HS } & 30 & $30.94 \pm 0.31$ & $78.51 \pm 0.62$ & $5.27 \pm 0.17$ & $32.57 \pm 0.52$ & $41.77 \pm 0.61$ & $10.44 \pm 0.52$ & $86.90 \pm 1.06$ & $2.34 \pm 0.07$ \\
\hline & 0 & 10 & $30.70 \pm 0.41$ & $80.71 \pm 1.05$ & $5.00 \pm 0.26$ & $34.98 \pm 0.81$ & $46.96 \pm 1.76$ & $11.24^{b} \pm 1.45$ & $89.15^{b} \pm 2.85$ & $2.36 \pm 0.02$ \\
\hline \multirow[t]{3}{*}{ TN } & 1 & 10 & $30.99 \pm 0.53$ & $79.53 \pm 0.89$ & $4.90 \pm 0.38$ & $34.39 \pm 1.60$ & $45.68 \pm 1.59$ & $10.51^{\mathrm{ab}} \pm 0.91$ & $87.38^{\mathrm{ab}} \pm 1.83$ & $2.38 \pm 0.03$ \\
\hline & 3 & 10 & $30.91 \pm 0.27$ & $77.96 \pm 0.73$ & $4.90 \pm 0.38$ & $33.88 \pm 1.28$ & $42.62 \pm 1.61$ & $9.75^{\mathrm{ab}} \pm 0.51$ & $86.75^{a b} \pm 1.00$ & $2.34 \pm 0.02$ \\
\hline & 0 & 10 & $30.50 \pm 0.45$ & $78.62 \pm 0.88$ & $5.20 \pm 0.36$ & $31.14 \pm 0.86$ & $42.48 \pm 1.07$ & $11.70^{b} \pm 0.74$ & $88.67^{b} \pm 1.48$ & $2.34 \pm 0.03$ \\
\hline \multirow[t]{2}{*}{ HS } & 1 & 10 & $30.91 \pm 0.55$ & $78.39 \pm 1.27$ & $5.40 \pm 0.27$ & $33.19 \pm 0.85$ & $40.94 \pm 0.78$ & $11.62^{b} \pm 0.84$ & $89.80^{b} \pm 1.56$ & $2.33 \pm 0.02$ \\
\hline & 3 & 10 & $31.40 \pm 0.63$ & $78.51 \pm 1.17$ & $5.20 \pm 0.25$ & $33.39 \pm 0.93$ & $41.90 \pm 1.32$ & $8.01^{a} \pm 0.64$ & $82.23^{a} \pm 1.58$ & $2.36 \pm 0.02$ \\
\hline
\end{tabular}


Table 4 Treatment means $( \pm S E)$ for effects of dietary supplementation with Alchemilla vulgaris and heat stress on egg quality traits that were expressed in late laying period during days 16 to 30 after the imposition of treatments

\begin{tabular}{|c|c|c|c|c|c|c|c|c|c|c|}
\hline \multicolumn{2}{|c|}{ Treatments $^{1}$} & $\mathrm{n}$ & $\begin{array}{l}\text { Egg weight } \\
\text { (gr) }\end{array}$ & $\begin{array}{l}\text { Eggshell weight } \\
\text { (gr) }\end{array}$ & $\begin{array}{l}\text { Eggshell } \\
\text { thickness } \\
\left(10^{-2} \mathrm{~mm}\right)\end{array}$ & $\begin{array}{c}\text { Albumen } \\
\text { height } \\
\text { (mm) }\end{array}$ & $\begin{array}{l}\text { Albumen length } \\
\qquad(\mathrm{mm})\end{array}$ & $\begin{array}{l}\text { Egg yolk height } \\
(\mathrm{mm})\end{array}$ & $\begin{array}{c}\text { Egg yolk } \\
\text { diameter }(\mathrm{mm})\end{array}$ & Egg width $(\mathrm{mm})$ \\
\hline \multicolumn{2}{|l|}{ TN } & 30 & $10.19 \pm 0.24$ & $0.90 \pm 0.03$ & $29.25 \pm 0.76$ & $3.75 \pm 0.15$ & $45.11 \pm 0.93$ & $9.30 \pm 0.16$ & $23.55 \pm 0.29$ & $24.39 \pm 0.18$ \\
\hline \multirow[t]{2}{*}{ HS } & & 30 & $9.63 \pm 0.15$ & $0.80 \pm 0.01$ & $28.52 \pm 0.70$ & $3.28 \pm 0.12$ & $42.71 \pm 0.61$ & $9.40 \pm 0.12$ & $24.50 \pm 0.25$ & $24.13 \pm 0.13$ \\
\hline & 0 & 10 & $10.07 \pm 0.52$ & $0.92^{b} \pm 0.06$ & $31.16 \pm 1.39$ & $3.50 \pm 0.21$ & $46.92 \pm 2.35$ & $9.24 \pm 0.33$ & $22.85 \pm 0.46$ & $24.27 \pm 0.40$ \\
\hline \multirow[t]{3}{*}{ TN } & 1 & 10 & $10.54 \pm 0.32$ & $0.88^{b} \pm 0.03$ & $29.48 \pm 0.73$ & $4.00 \pm 0.27$ & $44.45 \pm 1.04$ & $9.69 \pm 0.23$ & $24.45 \pm 0.48$ & $24.68 \pm 0.21$ \\
\hline & 3 & 10 & $9.97 \pm 0.40$ & $0.89^{b} \pm 0.05$ & $27.11 \pm 1.51$ & $3.74 \pm 0.27$ & $43.96 \pm 1.07$ & $8.97 \pm 0.24$ & $23.36 \pm 0.49$ & $24.22 \pm 0.33$ \\
\hline & 0 & 10 & $9.94 \pm 0.31$ & $0.84^{\mathrm{ab}} \pm 0.02$ & $28.96 \pm 1.05$ & $3.29 \pm 0.20$ & $42.98 \pm 1.19$ & $9.48 \pm 0.14$ & $23.81 \pm 0.57$ & $24.37 \pm 0.23$ \\
\hline \multirow[t]{3}{*}{ HS } & 1 & 10 & $9.49 \pm 0.29$ & $0.74^{\mathrm{a}} \pm 0.02$ & $27.86 \pm 1.46$ & $3.31 \pm 0.28$ & $43.41 \pm 0.93$ & $9.37 \pm 0.26$ & $23.42 \pm 0.44$ & $23.99 \pm 0.25$ \\
\hline & 3 & 10 & $9.47 \pm 0.19$ & $0.81^{\mathrm{ab}} \pm 0.03$ & $28.74 \pm 1.20$ & $3.23 \pm 0.16$ & $41.74 \pm 1.09$ & $9.35 \pm 0.25$ & $23.26 \pm 0.21$ & $24.04 \pm 0.18$ \\
\hline & & & $\begin{array}{l}\text { Egg length } \\
(\mathrm{mm})\end{array}$ & $\begin{array}{c}\text { Shape index } \\
(\%)\end{array}$ & $\begin{array}{l}\text { Egg yolk } \\
\text { colour }\end{array}$ & $\begin{array}{l}\text { Albumen width } \\
\qquad(\mathrm{mm})\end{array}$ & $\begin{array}{l}\text { Egg yolk } \\
\text { Index (\%) }\end{array}$ & $\begin{array}{l}\text { Albumen index } \\
(\%)\end{array}$ & Haugh units & $\begin{array}{l}\text { Shell strength } \\
\left(\mathrm{kg} / \mathrm{cm}^{2}\right)\end{array}$ \\
\hline \multicolumn{2}{|l|}{ TN } & 30 & $31.05 \pm 0.33$ & $78.69 \pm 0.58$ & $5.07 \pm 0.26$ & $31.97 \pm 0.62$ & $39.55 \pm 0.65$ & $9.81 \pm 0.42$ & $86.19 \pm 0.79$ & $2.36 \pm 0.02$ \\
\hline \multirow[t]{2}{*}{ HS } & & 30 & $30.32 \pm 0.22$ & $79.66 \pm 0.44$ & $4.93 \pm 0.28$ & $31.06 \pm 0.49$ & $40.08 \pm 0.56$ & $8.92 \pm 0.34$ & $83.82 \pm 0.77$ & $2.30 \pm 0.01$ \\
\hline & 0 & 10 & $30.93 \pm 0.69$ & $78.60 \pm 0.86$ & $5.40 \pm 0.34$ & $31.39 \pm 1.13$ & $40.45 \pm 1.26$ & $9.12 \pm 0.74$ & $84.92 \pm 1.07$ & $2.34 \pm 0.04$ \\
\hline \multirow[t]{3}{*}{ TN } & 1 & 10 & $31.47 \pm 0.53$ & $78.59 \pm 1.26$ & $5.30 \pm 0.49$ & $32.35 \pm 1.02$ & $39.69 \pm 0.86$ & $10.47 \pm 0.78$ & $87.30 \pm 1.62$ & $2.39 \pm 0.03$ \\
\hline & 3 & 10 & $30.74 \pm 0.50$ & $78.87 \pm 0.97$ & $4.50 \pm 0.50$ & $32.18 \pm 1.14$ & $38.52 \pm 1.21$ & $9.84 \pm 0.68$ & $86.34 \pm 1.41$ & $2.34 \pm 0.03$ \\
\hline & 0 & 10 & $30.59 \pm 0.50$ & $79.77 \pm 0.85$ & $4.30 \pm 0.40$ & $30.94 \pm 0.99$ & $39.96 \pm 0.84$ & $8.93 \pm 0.54$ & $83.67 \pm 1.16$ & $2.34 \pm 0.03$ \\
\hline \multirow[t]{2}{*}{ HS } & 1 & 10 & $30.36 \pm 0.40$ & $79.08 \pm 0.84$ & $5.10 \pm 0.43$ & $30.93 \pm 0.79$ & $40.10 \pm 1.18$ & $8.94 \pm 0.77$ & $84.02 \pm 1.82$ & $2.30 \pm 0.03$ \\
\hline & 3 & 10 & $30.01 \pm 0.20$ & $80.13 \pm 0.64$ & $5.40 \pm 0.56$ & $31.32 \pm 0.82$ & $40.19 \pm 0.98$ & $8.89 \pm 0.48$ & $83.76 \pm 1.02$ & $2.30 \pm 0.02$ \\
\hline
\end{tabular}


Table 5 Treatment means $( \pm S E)$ for effects of dietary supplementation with Alchemilla vulgaris and heat stress on egg quality traits that were expressed in late laying period during days 31 to 45 after the imposition of treatments

\begin{tabular}{|c|c|c|c|c|c|c|c|c|c|c|}
\hline \multicolumn{2}{|c|}{ Treatments $^{1}$} & \multirow{2}{*}{$\begin{array}{c}\mathrm{n} \\
30\end{array}$} & \multirow{2}{*}{$\begin{array}{c}\begin{array}{c}\text { Egg weight } \\
\text { (gr) }\end{array} \\
10.33 \pm 0.23\end{array}$} & \multirow{2}{*}{$\begin{array}{c}\begin{array}{c}\text { Eggshell weight } \\
\text { (gr) }\end{array} \\
0.87 \pm 0.02\end{array}$} & \multirow{2}{*}{$\begin{array}{c}\begin{array}{c}\text { Eggshell } \\
\text { thickness } \\
\left(10^{-2} \mathrm{~mm}\right)\end{array} \\
26.97 \pm 0.56\end{array}$} & \multirow{2}{*}{$\begin{array}{c}\begin{array}{c}\text { Albumen height } \\
(\mathrm{mm})\end{array} \\
3.72 \pm 0.11\end{array}$} & \multirow{2}{*}{$\begin{array}{c}\begin{array}{c}\text { Albumen length } \\
(\mathrm{mm})\end{array} \\
43.35 \pm 0.80\end{array}$} & \multirow{2}{*}{$\begin{array}{c}\begin{array}{c}\text { Egg yolk height } \\
(\mathrm{mm})\end{array} \\
9.34 \pm 0.11\end{array}$} & \multirow{2}{*}{$\begin{array}{c}\begin{array}{c}\text { Egg yolk } \\
\text { diameter }(\mathrm{mm})\end{array} \\
23.68 \pm 0.34\end{array}$} & \multirow{2}{*}{$\begin{array}{c}\text { Egg width (mm) } \\
24.43 \pm 0.17\end{array}$} \\
\hline TN & & & & & & & & & & \\
\hline HS & & 30 & $10.17 \pm 0.17$ & $0.89 \pm 0.02$ & $27.54 \pm 0.63$ & $3.49 \pm 0.13$ & $44.81 \pm 0.53$ & $9.29 \pm 0.09$ & $24.14 \pm 0.28$ & $24.39 \pm 0.13$ \\
\hline & 0 & 10 & $10.83 \pm 0.45$ & $0.98^{c} \pm 0.04$ & $28.62 \pm 0.92$ & $4.04^{c} \pm 0.14$ & $45.33^{b c} \pm 1.46$ & $9.46 \pm 0.27$ & $24.10^{b} \pm 0.63$ & $24.73 \pm 0.30$ \\
\hline \multirow[t]{3}{*}{$\mathrm{TN}$} & 1 & 10 & $10.53 \pm 0.27$ & $0.82^{\mathrm{ab}} \pm 0.03$ & $26.40 \pm 0.93$ & $3.56^{\mathrm{abc}} \pm 0.21$ & $43.69^{\mathrm{abc}} \pm 1.18$ & $9.39 \pm 0.11$ & $24.59^{b} \pm 0.42$ & $24.67 \pm .024$ \\
\hline & 3 & 10 & $9.63 \pm 0.40$ & $0.80^{\mathrm{a}} \pm 0.04$ & $25.90 \pm 0.93$ & $3.55^{\mathrm{abc}} \pm 0.20$ & $41.02^{\mathrm{a}} \pm 1.24$ & $9.17 \pm 0.16$ & $22.34^{a} \pm 0.51$ & $23.90 \pm 0.30$ \\
\hline & 0 & 10 & $9.95 \pm 0.31$ & $0.89^{b c} \pm 0.03$ & $29.38 \pm 1.03$ & $3.89^{b c} \pm 0.23$ & $42.59^{\mathrm{ab}} \pm 0.72$ & $9.42 \pm 0.17$ & $23.27^{\mathrm{a}} \pm 0.51$ & $24.17 \pm 0.18$ \\
\hline \multirow[t]{3}{*}{ HS } & 1 & 10 & $10.12 \pm 0.13$ & $0.85^{\mathrm{ab}} \pm 0.02$ & $26.69 \pm 1.24$ & $3.23^{\mathrm{a}} \pm 0.19$ & $46.28^{c} \pm 1.05$ & $9.03 \pm 0.13$ & $24.77^{\mathrm{b}} \pm 0.61$ & $24.45 \pm 0.15$ \\
\hline & 3 & 10 & $10.46 \pm 0.37$ & $0.92^{b c} \pm 0.03$ & $26.56 \pm 0.83$ & $3.35^{\mathrm{ab}} \pm 0.21$ & $45.57^{\mathrm{bc}} \pm 0.45$ & $9.41 \pm 0.14$ & $24.37^{b} \pm 0.31$ & $24.55 \pm 0.33$ \\
\hline & & & $\begin{array}{l}\text { Egg length } \\
(\mathrm{mm})\end{array}$ & $\begin{array}{c}\text { Shape index } \\
(\%)\end{array}$ & $\begin{array}{l}\text { Egg yolk } \\
\text { colour }\end{array}$ & $\begin{array}{l}\text { Albumen width } \\
\qquad(\mathrm{mm})\end{array}$ & $\begin{array}{l}\text { Egg yolk } \\
\text { Index (\%) }\end{array}$ & $\begin{array}{l}\text { Albumen index } \\
(\%)\end{array}$ & Haugh units & $\begin{array}{l}\text { Shell strength } \\
\left(\mathrm{kg} / \mathrm{cm}^{2}\right)\end{array}$ \\
\hline $\mathrm{TN}$ & & 30 & $31.46 \pm 0.33$ & $77.82 \pm 0.65$ & $4.70 \pm 0.29$ & $31.88 \pm 0.74$ & $39.59 \pm 0.52$ & $9.95 \pm 0.33$ & $85.96 \pm 0.65$ & $2.37 \pm 0.01$ \\
\hline \multirow[t]{2}{*}{ HS } & & 30 & $31.08 \pm 0.26$ & $78.56 \pm 0.54$ & $4.80 \pm 0.23$ & $31.84 \pm 0.46$ & $38.64 \pm 0.60$ & $9.18 \pm 0.39$ & $84.59 \pm 0.85$ & $2.36 \pm 0.01$ \\
\hline & 0 & 10 & $31.98 \pm 0.66$ & $77.50 \pm 1.14$ & $4.00 \pm 0.39$ & $33.83 \pm 1.31$ & $39.29^{\mathrm{abc}} \pm 0.68$ & $10.34 \pm 0.6$ & $87.51 \pm 0.81$ & $2.41 \pm 0.04$ \\
\hline \multirow[t]{3}{*}{ TN } & 1 & 10 & $31.79 \pm 0.35$ & $77.69 \pm 1.12$ & $5.00 \pm 0.33$ & $32.36 \pm 1.04$ & $38.33^{\mathrm{ab}} \pm 0.98$ & $9.37 \pm 0.6$ & $84.82 \pm 1.13$ & $2.39 \pm 0.02$ \\
\hline & 3 & 10 & $30.60 \pm 0.62$ & $78.28 \pm 1.22$ & $5.10 \pm 0.67$ & $29.45 \pm 1.15$ & $41.16^{c} \pm 0.84$ & $10.15 \pm 0.6$ & $85.53 \pm 1.30$ & $2.31 \pm 0.03$ \\
\hline & 0 & 10 & $30.67 \pm 0.46$ & $78.91 \pm 0.83$ & $4.60 \pm 0.37$ & $31.48 \pm 0.83$ & $40.57^{b c} \pm 0.90$ & $10.55 \pm 0.7$ & $87.21 \pm 1.42$ & $2.34 \pm 00.3$ \\
\hline \multirow[t]{2}{*}{ HS } & 1 & 10 & $31.25 \pm 0.45$ & $78.38 \pm 1.16$ & $5.00 \pm 0.47$ & $32.07 \pm 0.99$ & $36.68^{\mathrm{a}} \pm 1.139$ & $8.35 \pm 0.6$ & $83.06 \pm 1.31$ & $2.35 \pm 0.01$ \\
\hline & 3 & 10 & $31.33 \pm 0.44$ & $78.41 \pm 0.88$ & $4.80 \pm 0.38$ & $31.98 \pm 0.56$ & $38.67^{\mathrm{abc}} \pm 0.76$ & $8.64 \pm 0.5$ & $83.49 \pm 1.46$ & $2.38 \pm 0.03$ \\
\hline
\end{tabular}

${ }^{1} \mathrm{TN}$ : housed in temperature-controlled rooms at $22 \pm 2{ }^{\circ} \mathrm{C}$ for $24 \mathrm{~h} /$ day, $\mathrm{HS}: 34 \pm 2{ }^{\circ} \mathrm{C}$ for $8 \mathrm{~h} /$ day (between $09 \mathrm{~h} 00$ and $17 \mathrm{~h} 00$ ) and $22 \pm 2{ }^{\circ} \mathrm{C}$ for $16 \mathrm{~h} / \mathrm{day}, 0$ : unsupplemented, 1: supplemented with $1 \%$ Alchemilla vulgari powder, 3 : supplemented with $3 \%$ Alchemilla vulgari powder

$a, b, c$ Within a column, means with a common superscript do not differ with a probability of $P \leq 0.05$ 
Table 6 Treatment means ( \pm SE) for effects of dietary supplementation with Alchemilla vulgaris and heat stress on egg quality traits that were expressed in late laying period during days 46 to 60 after the imposition of treatments

\begin{tabular}{|c|c|c|c|c|c|c|c|c|c|c|}
\hline \multicolumn{2}{|c|}{ Treatments $^{1}$} & \multirow{2}{*}{$\begin{array}{l}\mathrm{n} \\
30\end{array}$} & \multirow{2}{*}{$\begin{array}{r}\text { Egg weight } \\
(\mathrm{gr})\end{array}$} & \multirow{2}{*}{$\begin{array}{r}\begin{array}{r}\text { Eggshell } \\
\text { weight } \\
(\mathrm{gr})\end{array} \\
0.87 \pm 0.02\end{array}$} & \multirow{2}{*}{$\begin{array}{r}\begin{array}{r}\text { Eggshell } \\
\text { thickness } \\
\left(10^{-2} \mathrm{~mm}\right)\end{array} \\
26.10 \pm 0.52\end{array}$} & \multirow{2}{*}{$\begin{array}{r}\begin{array}{r}\text { Albumen height } \\
(\mathrm{mm})\end{array} \\
3.64 \pm 0.12\end{array}$} & \multirow{2}{*}{$\begin{array}{r}\begin{array}{r}\text { Albumen length } \\
(\mathrm{mm})\end{array} \\
43.89 \pm 0.62\end{array}$} & \multirow{2}{*}{$\begin{array}{r}\begin{array}{r}\text { Egg yolk height } \\
(\mathrm{mm})\end{array} \\
9.15 \pm 0.14\end{array}$} & $\begin{array}{l}\text { Egg yolk diameter } \\
(\mathrm{mm})\end{array}$ & \multirow{2}{*}{$\begin{array}{l}\text { Egg width }(\mathrm{mm}) \\
\qquad 24.43 \pm 0.19\end{array}$} \\
\hline TN & & & & & & & & & $23.76 \pm 0.32$ & \\
\hline HS & & 30 & $10.03 \pm 0.21$ & $0.85 \pm 0.01$ & $25.89 \pm 0.50$ & $3.21 \pm 0.13$ & $41.61 \pm 1.44$ & $9.37 \pm 0.13$ & $23.64 \pm 0.26$ & $24.39 \pm 0.23$ \\
\hline & 0 & 10 & $9.89 \pm 0.48$ & $0.85 \pm 0.05$ & $25.79 \pm 0.94$ & $4.03^{b} \pm 0.23$ & $43.55 \pm 0.94$ & $9.41 \pm 0.17$ & $23.02 \pm 0.46$ & $24.07 \pm 0.36$ \\
\hline \multirow[t]{3}{*}{ TN } & 1 & 10 & $11.19 \pm 0.30$ & $0.93 \pm 0.03$ & $26.59 \pm 0.92$ & $3.40^{\mathrm{a}} \pm 0.20$ & $45.27 \pm 1.15$ & $9.22 \pm 0.20$ & $24.89 \pm 0.44$ & $25.10 \pm 0.22$ \\
\hline & 3 & 10 & $10.14 \pm 0.42$ & $0.83 \pm 0.03$ & $25.98 \pm 0.91$ & $3.49^{\mathrm{ab}} \pm 0.13$ & $43.06 \pm 1.08$ & $8.86 \pm 0.31$ & $23.51 \pm 0.60$ & $24.20 \pm 0.30$ \\
\hline & 0 & 10 & $10.00 \pm 0.40$ & $0.88 \pm 0.02$ & $26.27 \pm 0.84$ & $3.57^{\mathrm{ab}} \pm 0.15$ & $42.35 \pm 1.57$ & $9.47 \pm 0.23$ & $23.45 \pm 0.52$ & $24.27 \pm 0.29$ \\
\hline \multirow[t]{3}{*}{ HS } & 1 & 10 & $10.22 \pm 0.49$ & $0.81 \pm 0.04$ & $26.43 \pm 0.97$ & $3.03^{a} \pm 0.24$ & $44.23 \pm 1.97$ & $9.24 \pm 0.27$ & $24.06 \pm 0.55$ & $24.30 \pm 0.32$ \\
\hline & 3 & 10 & $9.93 \pm 0.23$ & $0.85 \pm 0.01$ & $25.13 \pm 0.83$ & $2.99^{\mathrm{a}} \pm 0.23$ & $39.03 \pm 3.26$ & $9.35 \pm 0.18$ & $23.55 \pm 0.28$ & $24.58 \pm 0.54$ \\
\hline & & & $\begin{array}{r}\text { Egg length } \\
(\mathrm{mm})\end{array}$ & $\begin{array}{r}\text { Shape index } \\
(\%)\end{array}$ & $\begin{array}{r}\text { Egg yolk } \\
\text { colour }\end{array}$ & $\begin{array}{r}\text { Albumen width } \\
(\mathrm{mm})\end{array}$ & $\begin{array}{l}\text { Egg yolk } \\
\text { Index (\%) }\end{array}$ & $\begin{array}{r}\text { Albumen index } \\
(\%)\end{array}$ & Haugh units & $\begin{array}{r}\text { Shell strength } \\
\left(\mathrm{kg} / \mathrm{cm}^{2}\right)\end{array}$ \\
\hline TN & & 30 & $31.37 \pm 0.35$ & $78.02 \pm 0.63$ & $4.93 \pm 0.26$ & $32.24 \pm 0.60$ & $38.73 \pm 0.82$ & $9.62 \pm 0.32$ & $85.46 \pm 0.69$ & $2.37 \pm 0.02$ \\
\hline \multirow[t]{2}{*}{ HS } & & 30 & $30.94 \pm 0.32$ & $78.94 \pm 0.79$ & $4.85 \pm 0.28$ & $33.14 \pm 0.56$ & $39.67 \pm 0.80$ & $8.80 \pm 0.48$ & $83.06 \pm 0.80$ & $2.34 \pm 0.02$ \\
\hline & 0 & 10 & $30.69 \pm 0.62$ & $78.56 \pm 1.02$ & $3.90^{\mathrm{a}} \pm 0.23$ & $32.94 \pm 0.54$ & $40.96 \pm 0.80$ & $10.57 \pm 0.65$ & $88.17^{c} \pm 1.19$ & $2.33 \pm 0.04$ \\
\hline \multirow[t]{3}{*}{$\mathrm{TN}$} & 1 & 10 & $32.38 \pm 0.38$ & $77.59 \pm 0.98$ & $4.44^{\mathrm{ab}} \pm 0.38$ & $33.33 \pm 1.08$ & $37.16 \pm 1.12$ & $8.67 \pm 0.50$ & $83.22^{\mathrm{ab}} \pm 1.26$ & $2.44 \pm 0.02$ \\
\hline & 3 & 10 & $31.16 \pm 0.66$ & $77.87 \pm 1.27$ & $6.27^{c} \pm 0.30$ & $30.70 \pm 1.21$ & $37.98 \pm 1.82$ & $9.52 \pm 0.41$ & $84.84^{\mathrm{abc}} \pm 0.71$ & $2.35 \pm 0.04$ \\
\hline & 0 & 10 & $30.72 \pm 0.48$ & $79.07 \pm 0.72$ & $3.90^{\mathrm{a}} \pm 0.28$ & $33.37 \pm 0.98$ & $40.44 \pm 0.78$ & $9.49 \pm 0.43$ & $85.43^{b c} \pm 0.83$ & $2.34 \pm 0.04$ \\
\hline \multirow[t]{2}{*}{ HS } & 1 & 10 & $31.23 \pm 0.80$ & $78.04 \pm 1.73$ & $5.57^{c} \pm 0.43$ & $33.37 \pm 1.17$ & $38.46 \pm 1.04$ & $7.95 \pm 0.83$ & $81.70^{\mathrm{a}} \pm 1.58$ & $2.36 \pm 0.04$ \\
\hline & 3 & 10 & $30.69 \pm 0.62$ & $78.56 \pm 1.02$ & $3.90^{\mathrm{a}} \pm 0.23$ & $32.94 \pm 0.54$ & $40.96 \pm 0.80$ & $10.57 \pm 0.65$ & $88.17^{c} \quad \pm 1.19$ & $2.33 \pm 0.04$ \\
\hline
\end{tabular}

${ }^{1} \mathrm{TN}$ : housed in temperature-controlled rooms at $22 \pm 2{ }^{\circ} \mathrm{C}$ for $24 \mathrm{~h} /$ day, $\mathrm{HS}: 34 \pm 2{ }^{\circ} \mathrm{C}$ for $8 \mathrm{~h} /$ day (between $09 \mathrm{h00}$ and $17 \mathrm{h00}$ ) and $22 \pm 2{ }^{\circ} \mathrm{C}$ for $16 \mathrm{~h} /$ day, 0 : unsupplemented, 1: supplemented with $1 \%$ Alchemilla vulgari powder, 3: supplemented with $3 \%$ Alchemilla vulgari powder

$a, b, c$ Within a column, means with a common superscript do not differ with a probability of $P \leq 0.05$ 
Table 7 Treatment means $( \pm \mathrm{SE}$ ) for effects of dietary supplementation with Alchemilla vulgaris and heat stress on egg quality traits that were expressed in late laying period during days 61 to 75 after the imposition of treatments

\begin{tabular}{|c|c|c|c|c|c|c|c|c|c|c|}
\hline \multicolumn{2}{|c|}{ Treatments $^{1}$} & $\mathrm{n}$ & $\begin{array}{l}\text { Egg weight } \\
\text { (gr) }\end{array}$ & $\begin{array}{l}\text { Eggshell } \\
\text { weight } \\
\text { (gr) }\end{array}$ & $\begin{array}{l}\text { Eggshell } \\
\text { thickness } \\
\left(10^{-2} \mathrm{~mm}\right)\end{array}$ & $\begin{array}{l}\text { Albumen } \\
\text { height } \\
(\mathrm{mm})\end{array}$ & $\begin{array}{l}\text { Albumen length } \\
\qquad(\mathrm{mm})\end{array}$ & $\begin{array}{c}\text { Egg yolk } \\
\text { height }(\mathrm{mm})\end{array}$ & $\begin{array}{l}\text { Egg yolk diameter } \\
\qquad(\mathrm{mm})\end{array}$ & Egg width $(\mathrm{mm})$ \\
\hline \multicolumn{2}{|l|}{ TN } & 30 & $10.11 \pm 0.23$ & $0.86 \pm 0.02$ & $25.90 \pm 0.40$ & $3.49 \pm 0.12$ & $43.53 \pm 0.68$ & $9.36 \pm 0.13$ & $23.43 \pm 0.29$ & $24.20 \pm 0.21$ \\
\hline \multirow[t]{2}{*}{ HS } & & 30 & $9.90 \pm 0.23$ & $0.86 \pm 0.01$ & $26.35 \pm 0.38$ & $3.29 \pm 0.12$ & $43.43 \pm 0.74$ & $9.25 \pm 0.13$ & $23.73 \pm 0.29$ & $24.21 \pm 0.17$ \\
\hline & 0 & 10 & $9.90 \pm 0.33$ & $0.84 \pm 0.03$ & $25.46 \pm 0.63$ & $3.57 \pm 0.24$ & $44.54^{\mathrm{abc}} \pm 0.87$ & $9.12 \pm 0.16$ & $23.29 \pm 0.49$ & $24.25 \pm 0.26$ \\
\hline \multirow[t]{3}{*}{ TN } & 1 & 10 & $10.55 \pm 0.39$ & $0.89 \pm 0.04$ & $25.51 \pm 0.68$ & $3.31 \pm 0.16$ & $45.14^{b c} \pm 1.25$ & $9.48 \pm 0.23$ & $23.78 \pm 0.53$ & $24.57 \pm 0.34$ \\
\hline & 3 & 10 & $9.92 \pm 0.46$ & $0.86 \pm 0.04$ & $26.69 \pm 0.75$ & $3.56 \pm 0.20$ & $41.08^{a} \pm 1.08$ & $9.49 \pm 0.28$ & $23.26 \pm 0.54$ & $23.82 \pm 0.47$ \\
\hline & 0 & 10 & $9.96 \pm 0.49$ & $0.90 \pm 0.03$ & $26.44 \pm 0.66$ & $3.45 \pm 0.19$ & $44.24^{\mathrm{abc}} \pm 1.05$ & $9.32 \pm 0.18$ & $24.15 \pm 0.53$ & $24.26 \pm 0.32$ \\
\hline \multirow[t]{3}{*}{ HS } & 1 & 10 & $10.25 \pm 0.47$ & $0.83 \pm 0.01$ & $27.35 \pm 1.03$ & $3.10 \pm 0.46$ & $45.88^{c} \pm 1.24$ & $8.83 \pm 0.46$ & $23.80 \pm 0.75$ & $24.50 \pm 0.48$ \\
\hline & 3 & 10 & $9.72 \pm 0.22$ & $0.83 \pm 0.01$ & $25.86 \pm 0.48$ & $3.20 \pm 0.13$ & $41.64^{\mathrm{ab}} \pm 1.16$ & $9.36 \pm 0.20$ & $23.29 \pm 0.34$ & $24.04 \pm 0.20$ \\
\hline & & & $\begin{array}{l}\text { Egg length } \\
(\mathrm{mm})\end{array}$ & $\begin{array}{l}\text { Shape index } \\
(\%)\end{array}$ & $\begin{array}{l}\text { Egg yolk } \\
\text { colour }\end{array}$ & $\begin{array}{l}\text { Albumen width } \\
\qquad(\mathrm{mm})\end{array}$ & $\begin{array}{l}\text { Egg yolk } \\
\text { Index (\%) }\end{array}$ & $\begin{array}{l}\text { Albumen } \\
\text { index (\%) }\end{array}$ & Haugh units & $\begin{array}{l}\text { Shell strength } \\
\left(\mathrm{kg} / \mathrm{cm}^{2}\right)\end{array}$ \\
\hline \multicolumn{2}{|l|}{$\mathrm{TN}$} & 30 & $30.79 \pm 0.46$ & $79.09 \pm 1.38$ & $3.93 \pm 0.22$ & $32.32 \pm 0.59$ & $40.08 \pm 0.68$ & $9.26 \pm 0.36$ & $84.70 \pm 0.77$ & $2.34 \pm 0.02$ \\
\hline \multirow[t]{2}{*}{ HS } & & 30 & $30.89 \pm 0.30$ & $78.47 \pm 0.59$ & $4.71 \pm 0.19$ & $32.02 \pm 0.60$ & $39.05 \pm 0.55$ & $8.75 \pm 0.32$ & $83.68 \pm 0.74$ & $2.33 \pm 0.02$ \\
\hline & 0 & 10 & $30.16 \pm 1.13$ & $81.49 \pm 3.44$ & $3.70^{\mathrm{a}} \pm 0.15$ & $31.86 \pm 1.29$ & $39.26 \pm 0.87$ & $9.35 \pm 0.61$ & $85.36 \pm 1.46$ & $2.33 \pm 0.03$ \\
\hline \multirow[t]{3}{*}{ TN } & 1 & 10 & $31.66 \pm 0.45$ & $77.66 \pm 0.97$ & $3.78^{\mathrm{a}} \pm 0.40$ & $32.86 \pm 0.94$ & $40.00 \pm 1.23$ & $8.57 \pm 0.58$ & $83.25 \pm 1.23$ & $2.39 \pm 0.04$ \\
\hline & 3 & 10 & $30.63 \pm 0.58$ & $77.97 \pm 1.91$ & $4.30^{a} \pm 0.52$ & $32.30 \pm 0.85$ & $40.96 \pm 1.43$ & $9.79 \pm 0.65$ & $85.36 \pm 1.29$ & $2.33 \pm 0.04$ \\
\hline & 0 & 10 & $31.10 \pm 0.69$ & $78.19 \pm 1.06$ & $4.40^{\mathrm{ab}} \pm 0.31$ & $32.35 \pm 0.75$ & $38.73 \pm 1.00$ & $9.04 \pm 0.52$ & $84.65 \pm 1.27$ & $2.34 \pm 0.04$ \\
\hline \multirow[t]{2}{*}{ HS } & 1 & 10 & $31.18 \pm 0.20$ & $78.61 \pm 1.76$ & $5.50^{b} \pm 0.29$ & $34.83 \pm 2.10$ & $37.01 \pm 0.90$ & $7.74 \pm 1.21$ & $82.07 \pm 2.52$ & $2.36 \pm 0.04$ \\
\hline & 3 & 10 & $30.56 \pm 0.25$ & $78.70 \pm 0.77$ & $4.70^{\mathrm{ab}} \pm 0.30$ & $30.56 \pm 0.67$ & $40.19 \pm 0.66$ & $8.88 \pm 0.36$ & $83.36 \pm 0.84$ & $2.32 \pm 0.02$ \\
\hline
\end{tabular}




\section{Discussion}

The present study was conducted to investigate the effects of supplemental AV on egg production and egg quality during the late laying period of quails that were exposed to high environmental temperature. In developing countries, eggs are one of the most consumed foods of animal origin because of their low cost, reliability and healthfulness (Neumann et al., 2002; Ndlovu, 2010).

It has been reported that high ambient temperature causes significant deterioration of egg quality and results in reduced performance in poultry production (Smith, 1974; Wolfenson et al., 1979; Emery et al., 1984; Yalcin et al., 2001; Sahin \& Kucuk, 2003). Here, adaption of quail to heat stress is made apparent by the lack of significant effects that were observed after the initial 15 days.

Dietary AV is thought to have huge potential to improve egg laying performance and indicators of egg quality. Indeed, in addition to those fundamental effects of dietary AV, it is reported that there are significant therapeutic roles in female reproduction problems (Spiridonov et al., 2005; Saric-Kundalic et al., 2011). In contrast to the results of Saric-Kundalic et al. (2011), dietary supplementation with AV did not increase the laying performance of quail reared at TN conditions. In agreement with the literature, lower egg production, feed consumption, and egg weight and increased FCR were observed for quail that were exposed to HS. In the present study, dietary AV was found to reduce egg production in TN conditions. However, at $3 \%$ of the diet, AV supplementation of quail that were housed under HS conditions prevented the reduced egg production and FCR that were observed under TN conditions.

To the best of the authors' knowledge, this is the first study to examine the effect of dietary AV on laying performance in the late laying period of heat-stressed quails. The present results in heat-stress quail are thought to be related to the effects of high antioxidant ingredients such as flavonoids (D'Agostino et al., 1998) that are found in AV. It has been shown that flavonoids exhibit antioxidant effects (Jovanovic et al., 1994; Fraga, 2010), and improve the performance of stressed animals (Ting et al., 2011; Surai, 2013). This may be because of the improved efficacy of antioxidant agents in challenging conditions, as postulated by Akbarian et al. (2016). Liu et al. (2013, 2014) and Iskender et al. (2017) found similar results to those of the present study, in which egg weight and feed intake were not changed by dietary flavonoids in laying hens housed under TN conditions. In contrast, it has been found that supplementation with the flavonoid quercetin reduced FCR and increased egg quality and production in a dose-dependent manner during the late laying period (Liu et al., 2013). It is emphasized that flavonoids, which have no effect on birds that are housed under TN conditions, may show antioxidant activity in the presence of a stressor such as heat and that the response may be dose dependent (Galati \& O'Brien, 2004; Akbarian et al., 2016; Iskender et al., 2017).

It is generally known that performance and egg quality parameters of birds deteriorate during the late laying period (Rodriguez-Navarro et al., 2002). This deterioration may be worsened by oxidative stress that arises from high environmental temperatures. In the present study, various indicators indicated a degradation of egg quality over time in quail that were housed in HS conditions. Numerous studies have been conducted to examine the potential for dietary supplementation to mitigate effects of heat stress in hens (Sahin \& Kucuk, 2003). Dietary supplementation with AV has also shown a lack of effect on egg quality of various flavonoid compounds (hesperidin, naringin and quercetin) supplemented to layers that are reared under TN conditions (Yildiz et al., 2006; Liu et al., 2014; Iskender et al., 2017).

Although HS significantly reduced eggshell weight until day 45 , dietary supplementation with AV improved it afterwards and it was maintained throughout the remainder of the present experiment. In agreement with the present data, other researchers observed no change in the eggshell thickness and eggshell strength because of dietary supplementation with flavonoids such as hesperidin, naringin and quercetin (Lien et al., 2008; Ting et al., 2011; Goliomytis et al., 2014; Iskender et al., 2017). However, there have been observations of increased eggshell thickness and strength owing to such supplementation of quail (Ni et al., 2007; Sahin et al., 2007; Liu et al., 2013). Heat stress increases the blood pH and causes respiratory alkalosis, resulting in decreased calcium (Ca) absorption and less effective use of it (Sahin \& Kucuk, 2003; Franco-Jimenes et al., 2007; Ma et al., 2014). Fouad et al. (2016) reported that some phytochemicals that have antioxidant and oestrogenic properties, such as flavonoids, may improve the bioavailability of $\mathrm{Ca}$ and phosphorus $(\mathrm{P})$ and improve eggshell formation, thereby enhancing the production of normal quality eggs under HS. However, no effects of supplementation with AV on shell thickness and shell strength were observed in this study. Cassidy (2003) also stated that because of low amounts of oestrogen flavonoids prevent the absorption and use of $\mathrm{Ca}$ by executing an antagonistic activity during the late laying period.

\section{Conclusion}

In conclusion, the present study indicated that the high environmental temperature initially caused negative effects on egg production and egg quality, whereas dietary AV supplementation at $3 \%$ levels partially offset these effects in the late laying period of heat-stressed quails. Thus, supplementation with AV 
may be useful to at least partially prevent the decrease in egg production and the deterioration in egg quality during the late laying period of heat-stressed quails. However, further studies are needed to explain the effects of dietary AV on performance traits that were not measured in the present study and on egg quality.

\section{Acknowledgements}

The authors would like to thank O. Ordu and Y.S. Özdemir for their contributions to the current study.

\section{Authors' Contributions}

IS and AK performed the project and designed the study, AK and FA performed the experiments, AK and FA collected the samples and analysed them, IS did the statistical analysis, and FA wrote the paper.

\section{Conflict of Interest Declaration}

The authors declare that there are no conflicts of interest.

\section{References}

Afanas'ev, I.B., Dorozhko, A.I., Brodskii, A.V., Kostyuk, V.A. \& Potapovitch, A.I., 1989. Chelating and free scavenging mechanisms of inhibitory action of rutin and quercetin in lipid peroxidaton. Biochem. Pharmacol. 38,1763-1769.

Akbarian, A., Michiels, J., Degroote, J., Majdeddin, M., Golian, A. \& De Smet, S., 2016. Association between heat stress and oxidative stress in poultry; mitochondrial dysfunction and dietary interventions with phytochemicals. J. Anim. Sci. Biotechnol. 7, 37-51.

Akdemir, F. \& Sahin, K., 2009. Genistein supplementation to the quail: Effects on egg production and egg yolk genistein, daidzein, and lipid peroxidation levels. Poult. Sci. 88, 2125-2131.

Akdemir, F., Sahin, N., Orhan, C., Tuzcu, M., Sahin, K. \& Hayirli, A., 2015. Chromium-histidinate ameliorates productivity in heat-stressed Japanese quails through reducing oxidative stress and inhibiting heat-shock protein expression. Br. Poult. Sci. 56, 247-254.

AOAC, 1990. Official methods of analysis of the Association of Official Analytical Chemists (15th ed.). Washington DC, USA.

Baer, J., Lansford, R. \& Cheng, K., 2015. Chapter 22. Japanese quail as a laboratory animal model. In: J.G. Fox, L.C. Anderson, G.M. Otto, K.R. Pritchett-Corning \& M.T. Whary (eds.). Laboratory Animal Medicine 3rd edition. Academic Press, Elsevier B.V., Amsterdam, The Netherlands.

Cassidy, A., 2003. Potential risks and benefits of phytoestrogen-rich diets. Int. J. Vitam. Nutr. Res. 73: 120-126.

D'Agostino, M., Dini, I., Ramundo, E. \& Senatore, F., 1998. Flavonoid glycosides of Alchemilla vulgaris L. Phytother. Res. 12, 162-163.

Eisen, E.J., Bohren, B.B. \& McKean, H.E., 1962. The Haugh unit as a measure of egg albumen quality. Poult. Sci. 41, 1461-1468.

Emery, D.A., Vohra, P., Ernst, R.A. \& Morrison, S.R., 1984. The effect of cyclic and constant ambient temperatures on feed consumption, egg production, egg weight, and shell thickness of heat. Poult Sci. 63, 2027-2035.

Fouad, A.M., Chen, W., Ruan, D., Wang, S., Xia, W.G. \& Zheng, C.T., 2016. Impact of heat stress on meat, egg quality, immunity and fertility in poultry and nutritional factors that overcome these effects: A Review. Int. J. Poult. Sci. 15, 81-95.

Fraga, C.G., 2010. Plant phenolics and human health, biochemistry, nutrition and pharmacology. John Wiley \& Sons Inc. New Jersey, USA.

Franco-Jimenez, D.J., Scheideler, S.E., Kittok, R.J., Brown-Brandl, T.M., Robeson, L.R., Taira, H. \& Beck, M.M., 2007. Differential effects of heat stress in three strains of laying hens. J. Appl. Poult. Res. 16, 628-634.

Galati, G., \& O'Brien, P.J., 2004. Potential toxicity of flavonoids and other dietary phenolics: Significance for their chemopreventive and anticancer properties. Free Radic. Biol. Med. 37, 287-303.

Goliomytis, M., Orfanou, H., Petrou, E., Charismiadou, M.A., Simitzis, P.E. \& Deligeorgis, S.G., 2014. Effect of hesperidin dietary supplementation on hen performance, egg quality and yolk oxidative stability. Br. Poult. Sci. 55, 98-104.

Hanasaki, Y., Ogawa, S. \& Fukui, S., 1994. The correlation between active oxygen scavenging and antioxidative effects of flavonoids. Free Radic. Biol. Med. 16, 845-850.

Iskender, H., Yenice, G., Dokumacioglu, E., Kaynar, O., Hayirli, A. \& Kaya, A., 2017. Comparison of the effects of dietary supplementation of flavonoids on laying hen performance, egg quality and egg nutrient profile, Br. Poult. Sci. 58, 550-556.

Jovanovic, S.V., Steenken, S., Tosic, M., Marjanovic, B. \& Simic, M.G., 1994. Flavonoids as antioxidants. J. Am. Chem. Soc. 116, 4846-4851.

Jurgens, M.H., 1996. Animal feeding and nutrition (8th ed.). Kendall/Hunt. Dubuque, IA.

Kahkonen, M.P., Hopia, A.I., Vuorela, H.J., Rauha, J., Pihlaja, K., Kujala, T.S. \& Heinonen, M., 1999. Antioxidant activity of plant extracts containing phenolic compounds. J. Agric. Food Chem. 47, 3954-3962.

Kiselova, Y., Ivanova, D., Chervenkov, T., Gerova, D., Galunska, B. \& Yankova, T., 2006. Correlation between the in vitro antioxidant activity and polyphenol content of aqueous extracts from Bulgarian herbs. Phytother. Res. 20, 961-965.

Leeson, S., 1986. Nutritional considerations of poultry during heat stress. World's Poultry Science Journal 42, 69-81. 
Lien, T.F., Yeh, H.S. \& Su, W.T., 2008. Effect of adding extracted hesperetin, naringenin and pectin on egg cholesterol, serum traits and antioxidant activity in laying hens. Arch. Anim. Nutr. 62, 33-43.

Liu, H.N., Liu, Y., Hu, L.L., Suo, Y.L., Zhang, L., Jin, F.X., Feng, A., Teng, N. \& Li, Y., 2014. Effects of dietary supplementation of quercetin on performance, egg quality, cecal microflora populations, and antioxidant status in laying hens. Poult. Sci. 93, 347-353.

Liu, Y., Li, L., Liu, H.N., Suo, Y.L., Hu, L.L., Feng, X., Zhang, A.L. \& Jin, F., 2013. Effect of quercetin on performance and egg quality during the late laying period of hens. Br. Poult. Sci. 54, 510-514.

Lu, Q., Wen, J. \& Zhang, H., 2007. Effect of chronic heat exposure on fat deposition and meat quality in two genetic types of chicken. Poult. Sci. 86, 1059-1064.

Ma, X., Lin, Y., Zhang, H., Chen, W., Wang, S., Ruan, D. \& Jiang, Z., 2014. Heat stress impairs the nutritional metabolism and reduces the productivity of egg-laying ducks. Anim. Reproduc. Sci.145, 182-190.

Nair, M.P., Mahajan, S., Reynolds, J.L., Aalinkeel, R., Nair, H., Schwartz, S.A. \& Kandaswami, C., 2006. The flavonoid quercetin inhibits proinflammatory cytokine (tumor necrosis factor alpha) gene expression in normal peripheral blood mononuclear cells via modulation of the NF-kappabeta system. Clin. Vaccine Immunol. 13, 319-328.

Ndlovu, L., 2010. The role of foods of animal origin in human nutrition and health. In Swanepoel, F.J.C., Stroebel, A. \& Moyo, S., (eds). The role of livestock in developing communities: Enhancing multifunctionality. CTA, Wageningen, The Netherlands. pp. 77-90.

Neagu, E., Paun, G., Albu, C. \& Radu, G.L., 2015. Assessment of acetylcholinesterase and tyrosinase inhibitory and antioxidant activity of Alchemilla vulgaris and Filipendula ulmaria extracts. J. Taiwan Inst. Chem. Eng. 52: 1-6.

Neumann, C., Harris, D.M. \& Rogers, L.M., 2002. Contribution of animal source food in improving diet quality and function in children in the developing world. Nutr. Res. 22, 193-220.

Ni, Y.D., Zhu, Q., Zhou, Z.L., Grossmann, R., Chen, J. \& Zhao, R.Q., 2007. Effect of dietary daidzein on egg production, shell quality, and gene expression of ER-r, GH-R, and IGFIR in shell glands of laying hens. J. Agric. Food Chem. $55,6997-7001$.

Oktyabrskay, O., Vysochina, G., Muzyka, N., Samoilova, Z., Kukushkina, T. \& Smirnova, G., 2009. Assessment of antioxidant activity of plant extracts using microbial test systems. J. Appl. Microbiol. 106, 1175-1183.

Reddish, J.M., Nestor, K.E. \& Lilburn, M.S., 2003. Effect of selection for growth on onset of sexual maturity in randombred and growth-selected lines of Japanese quail. Poult. Sci. 82,187-191.

Rice-Evans, C., Miller, N. \& Paganga, G., 1997. Antioxidant properties of phenolic compounds. Trends Plant Sci. 2: 152159.

Rodriguez-Navarro, A., Kalin, O., Nys, Y. \& Garcia-Ruiz, J.M., 2002. Influence of the microstructure on the shell strength of eggs laid by hens of different ages. Br. Poult. Sci. 43, 395-403.

Ruskin, F.R., 1991. Micro-Livestock. National Academies Press. Washington DC, USA.

Sahin, K. \& Kucuk, O., 2003. Heat stress and dietary vitamin supplementation of poultry diets. Nutrition Abstracts and Reviews Series B: Livestock Feeds and Feeding 73, 41-50.

Sahin, N., Onderci, M., Sahin, K. \& Kucuk, O., 2008b. Supplementation with organic or inorganic selenium in heatdistressed quail. Biol Trace Elem. Res. 122, 229-237.

Sahin, N., Orhan, C., Tuzcu, M., Sahin, K. \& Kucuk, O., 2008a. The effects of tomato powder supplementation on performance and lipid peroxidation in quail. Poult. Sci. 87, 276-283.

Sahin, K., Akdemir, F., Orhan, C., Tuzcu, M., Hayirli, A. \& Sahin, N., 2010. Effects of dietary resveratrol supplementation on egg production and antioxidant status. Poult. Sci. 89, 1190-1198.

Sahin, N., Onderci, M., Balci, T.A., Cikim, G., Sahin, K. \& Kucuk, O., 2007. The effect of soy isoflavones on egg quality and bone mineralisaton during the late laying period of quail. Br. Poult. Sci. 48, 363-369.

Saric-Kundalic, B., Dobes, C., Klatte-Asselmeyer, V. \& Saukel, J., 2011. Ethnobotanical survey of traditionally used plants in human therapy of east, north and north-east Bosnia and Herzegovina. J. Ethnopharm. 133, 1051-1076.

Shane, S.M., 1988. Factors influencing health and performance of poultry in hot climates. Critic. Rev. Poult. Biol. 1, 247267.

Smith, A.J., 1974. Changes in the average weight and shell thickness of eggs produced by hens exposed to high environmental temperatures. A review. Trop. Anim. Health Prod. 6, 237-244.

Spiridonov, N.A., Konovalov, D.A. \& Arkhipov, V.V., 2005. Cytotoxicity of some Russian ethnomedicinal plants and plant compounds. Phytother. Res. 19, 428-432.

Surai, P.F., 2013. Polyphenol compounds in the chicken/animal diet: From the past to the future. J. Anim. Physiol. Anim. Nutr. 98, 19-31.

Ting, S., Yeh, H.S. \& Lien, T.F., 2011. Effects of supplemental levels of hesperetin and naringenin on egg quality, serum traits and antioxidant activity of laying hens. Anim. Feed Sci. Technol. 163, 59-66.

Tunsaringkarn, T., Tungjaroenchai, W. \& Siriwong, W., 2013. Nutrient benefits of Quail (Coturnix coturnix japonica) eggs. Int. J. Sci. Res. Publ. 3, 1-8.

Wolfenson, D., Feri, Y.F., Snapir, N. \& Berman, A., 1979. Effect of diurnal or nocturnal heat stress on egg formation. Br. Poult. Sci. 20, 167-174.

Yalcin, S., Ozkan, S., Turkmut, L. \& Siegel, P.B., 2001. Responses to heat stress in commercial and local broiler stocks. 1. Performance traits. Br. Poult. Sci. 42, 149-152.

Yildiz, G., Sacakli, P. \& Gungor, T., 2006. The effect of dietary Jerusalem artichoke (Helianthus tuberosus L.) on performance, egg quality characteristics and egg cholesterol content in laying hens. Can. J. Anim. Sci. 51, 349354. 